\title{
ESTUDO DA AVALIAÇÃO ESTRUTURAL DE UM CHASSIS UTILIZANDO O MÉTODO DE ELEMENTOS FINITOS
}

Marcos Antônio Neves Mesquita Filho1; Leo Guedes Blanco²; Lucas Aragão de Assis³; Pedro Bancillon Ventin Muniz ${ }^{4}$

1SENAI CIMATEC; Salvador/Bahia; marcosmesquita37@gmail.com

2 SENAI CIMATEC; Salvador/Bahia; leoguedesblanco@gmail.com

3 SENAI CIMATEC; Salvador/Bahia; I.aragao@hormail.com

4 SENAI CIMATEC; Salvador/Bahia; Pedro.muniz@fieb.org.br

Resumo: A análise pelo método de elementos finitos é comumente utilizado para prever o comportamento mecânico de estruturas quando submetidas a variados tipos de esforços, pois ela permite encontrar boas aproximações de soluções que não seriam viáveis por meios analíticos. Em um projeto para avaliação estrutural de um chassi de mini baja, esta ferramenta mostra-se necessária, devido a uma alta complexidade na geometria, e uma natureza imprevisível dos esforços que atuam na estrutura. Este trabalho descreve o estudo para avaliação estrutural do chassi, por meio de simulações em elementos finitos, usando o SolidWorks a fim de determinar sua rigidez torcional e, consequentemente, prever seu desempenho na competição.

Palavras-Chave: Rigidez Torcional; Baja; Chassi; Elementos Finitos

\section{STUDY OF THE STRUCTURAL VALIDATION OF A CHASSIS USING THE FINITE ELEMENTS' METHOD}

Abstract: The analysis by the finite elements' method is commonly used to predict the mechanical behavior of structures, because it allows to find good approximations of solutions that wouldn't be possible by analytic ways. In a project for the structural validation of a mini Baja's chassis, this tool has shown to be necessary, due to the high geometry complexity and an unpredictable nature of the loads that act on the structure. This work describes the study for the structural validation of the chassis by computational simulations in finite elements' method using the SolidWorks software, in order to determine its torsional stiffness and, consequently, predict its performance at the competition.

Keywords: Torsional Stiffness; Baja; Chassis; Finite Elements 


\section{Introdução}

Um chassi é considerado a espinha dorsal de um veículo, além da célula de sobrevivência do piloto, cujo nome vem do Latim "capsum", que significa caixa [1]. Ou seja, trata-se de uma gaiola grande o suficiente para comportar e proteger todos os demais componentes de um veículo e deve ser rígido e resistente o suficiente para transferir e absorver cargas durante o percurso, mantendo toda integridade do veículo. Outros requisitos são o de promover a proteção estrutural contra capotamentos e colisões, além de possuir características ergonômicas.

Analisando as suas funções, um projeto de chassis deverá levar em consideração algumas funções básicas a respeito do mesmo, que seriam: Apoiar e proteger o piloto, além dos demais componentes do veículo, resistindo a qualquer carregamento ao longo do percurso.

O chassi é um dos principais componentes de um veículo mini baja, ao qual consiste em um veículo off road que comporta apenas o piloto, construído por uma equipe de estudantes, tendo como objetivo participar de diversas provas, tanto estáticas quanto dinâmicas. Durante as provas dinâmicas, um chassi de mini baja passa por condições extremas de carregamento, tornando mais críticas as funções já listadas antes. Além disso, a competição possui um regulamento, no qual lista uma série de requisitos a serem seguidos, tais como membros obrigatórios (ver figura 1), medidas limite e entre outros.

Figura 1: Membros obrigatórios em um chassi

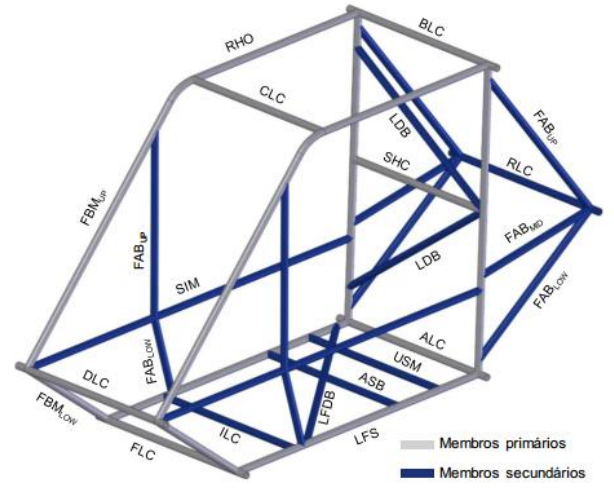

Fonte: Regulamento administrativo e técnico baja sae brasil

Para um projeto de chassis, algumas técnicas de otimização podem ser baseadas em 2 objetivos, são eles: Redução de massa: Para qualquer componente, quanto menor a massa, menores as propriedades inerciais, os carregamentos sofridos e a necessidade de reforços estruturais. Esta prática leva a um melhor desempenho quando analisado o quesito "aceleração X Peso". E Aumento da rigidez: para um chassi, a rigidez é responsável por garantir a estabilidade do veículo na pista, 
respeitando todos os posicionamentos dos componentes, as medidas ergonômicas que garantem o conforto e a segurança do piloto, e o trabalho ideal da suspensão [2].

Para realizar o validamento de um desenvolvimento de estrutura de chassis são utilizados softwares de análise de elementos finitos, através de simulações computacionais que geram esforços em determinados componentes, nos quais representam situações em pista. Neste artigo, foi utilizado o SolidWorks 2018 para as simulações estruturais, sendo abordada uma análise direcionada para componentes estruturais que visa determinar rigidez torcional do chassi.

A rigidez torcional, é a resistência de um corpo à deformação, quando este é submetido a um momento ao longo do seu comprimento longitudinal [2]. Esta característica, determina o comportamento do chassi ao percorrer curvas ou desviar de obstáculos. Ela é medida dividindo o torque aplicado na suspensão dianteira, com a traseira fixada, pelo ângulo gerado, determinando um valor que será constante para o regime das pequenas deformações. A rigidez torcional pode ser medida, tanto em uma bancada de testes, usando o chassi real, ou por meio virtual, usando o modelo CAD do chassi em programas de simulação estrutural. A vantagem da medição em CAD é reduzir consideráveis custos com tempo de manufatura e a aquisição de materiais. De acordo com estudos, existe uma faixa de valores ideias (ver Tabela 1) referentes à rigidez torcional $[1,3]$. Devido ao exposto, o foco deste artigo está em simulações estruturais por meios computacionais que irão auxiliar os especialistas a determinar um projeto estrutural de veiculo mini BAJA que contenha atributos necessários para o seu funcionamento e operação.

Tabela 1: Valores recomendados de rigidez torcional para diferentes veículos

\begin{tabular}{ll}
\hline Veículo & Rigidez Torcional (Nm/grau) \\
\hline Baja SAE & $750-1500$ \\
Fórmula SAE & $1000-5000$ \\
Carros de Passeio & $5000-25000$ \\
Carros de Corrida Nascar & $15000-30000$ \\
Carros Esportivos & $15000-40000$ \\
Fórmula 1 & $10000-100000$ \\
\hline
\end{tabular}

Fonte: Adaptado de (BARBOSA, 2015) e (SAMPÓ, 2011)

\section{METODOLOGIA}

A determinação de uma rigidez satisfatória para um chassi é vital no projeto, segundo Sampó, "O fato de que a rigidez torcional é o objetivo mais difícil de se obter durante o projeto, justifica o fato de que a rigidez de um chassi é frequentemente representada apenas pela rigidez torcional".

Portanto, é possível entender que, ao atingir um nível satisfatório de rigidez torcional, as demais características estruturais terão sido obtidas. A análise foi elaborada tomando como base o modelo Mini BAJA 2018, o qual apresentou diversos problemas durante provas dinâmicas de pista. Os principais pontos de estudo e 
modificações foram os membros ligados aos olhais dos amortecedores dianteiros e os membros que compõem o suporte do sistema de transmissão. É entendido que estes sejam os membros nos quais sofrem os maiores esforços durante a operação do veículo.

Visando garantir um desempenho satisfatório, os olhais de fixação dos amortecedores foram posicionados em componentes que possuem união de 1 ou mais membros estruturais, de maneira a se configurar um nó de uma treliça. Esta técnica visou impedir que esforços de flexão fossem gerados em membros do chassis, provocando falhas do amortecedor (ver figura 2) e no escoamento do membro estrutural nesta região.

Figura 2: Flecha formada no membro SIM devido esforço excessivo do amortecedor

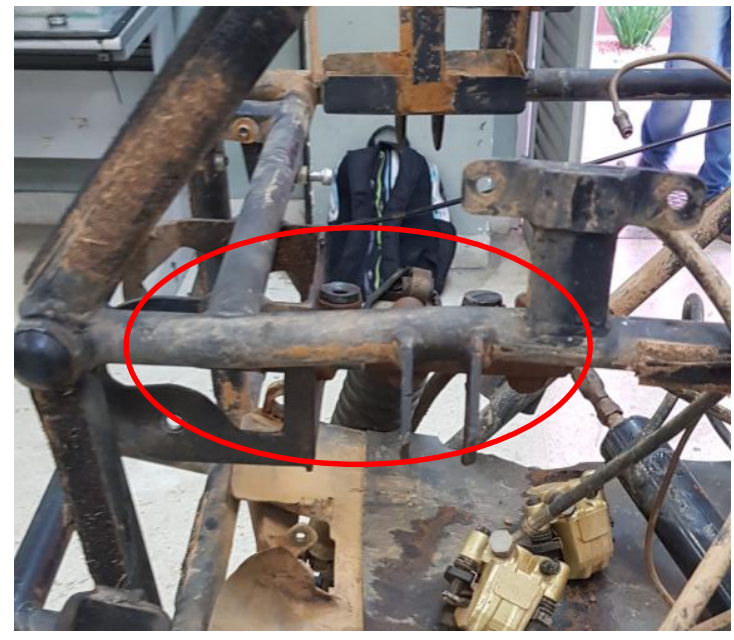

Para a outra região crítica em questão - o suporte do sistema de transmissão - foram tomadas algumas medidas, a fim de simplificar a estrutura e corrigir uma falha estrutural que surgiu durante os testes do protótipo de 2018.

Seguindo a orientação de Norton (2013) "Apenas seções circulares fechadas, tanto ocas como maciças, são recomendadas para aplicações com carregamento torcional.", e observando a configuração da estrutura antiga, onde o motor e a caixa de redução estavam suportados por uma chapa, soldada a dois tubos quadrados (ver Figura 4), provocando uma carga torcional no metalon e, consequentemente, ocasionando em uma fissura.

Agora o motor é apoiado, diretamente sobre dois tubos quadrados, facilitando sua montagem e gerando apenas uma carga de flexão, e a caixa de redução será suportada por olhais soldados em tubos (Figura 3). 
Figura 3: Fundo e posicionamento de Powertrain

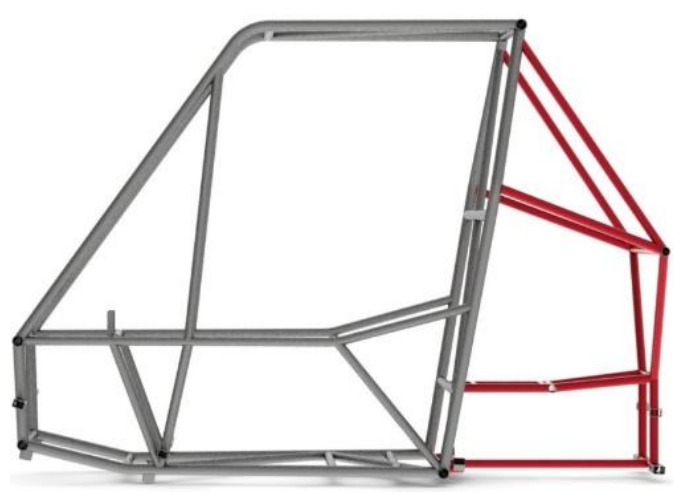

Figura 4: Fissura encontrada no suporte antigo

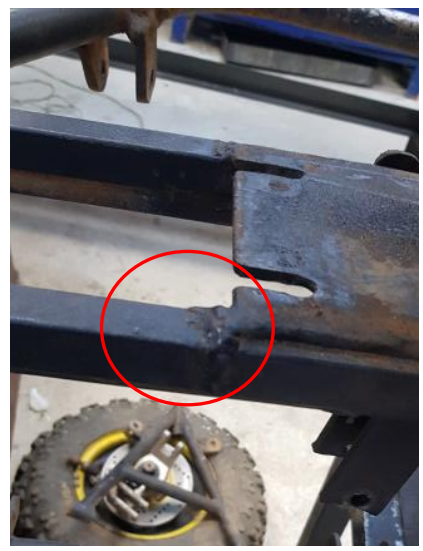

Demais membros como: SHC, FAB up e o SIM foram realocados para se enquadrar à regra da competição. O fundo do protótipo foi refeito, para alocar a nova geometria de suspensão e possibilitando o posicionamento mais baixo do sistema de transmissão. Além disso, alguns tubos foram retirados, tais como membros que uniam o LFS e o SIM, e o membro que unia o $\mathrm{RHO}$ e o $\mathrm{RRH}$. As abas de fixação do assoalho e firewall foram retiradas, e eles serão presos no atual veículo através de rebites nos próprios tubos da estrutura. As modificações serão mostradas a seguir.

- Reposicionamento do membro SHC (ver Figura 1)

- Reposicionamento do FABup dianteiro (ver Figura 1) - No protótipo 2018, o membro não respeitava a distância máxima de $127 \mathrm{~mm}$ medida verticalmente entre a interseção do FABup e o ponto C no RHO [5].

- Reposicionamento do SIM - No protótipo 2018 o membro RRH estava em desacordo com o item B6.2.2.5.4 [5], pois possuía um comprimento maior que $838 \mathrm{~mm}$ entre pontos denominados. Para enquadrá-lo à regra, o SIM passou a ser curvado, e unido ao $\mathrm{RRH}$ alguns milímetros acima da sua posição atual. Desta forma, o membro $\mathrm{RRH}$ passou a ter $791 \mathrm{~mm}$ entre os pontos denominados B e S.

Figura 5: Membro SIM

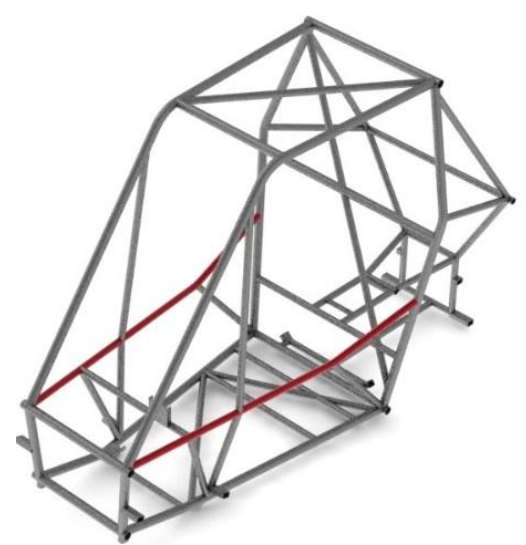


- Abas de fixação - Todos os suportes de fixação do assoalho e do firewall foram retirados, para facilitar a manufatura e reduzir massa do chassi. $O$ assoalho terá sua espessura reduzida, e será feito com chapa de alumínio de $0,5 \mathrm{~mm}$. Assoalho e firewall serão presos diretamente nos tubos da gaiola através de rebites de repuxo.

Após estas considerações, novo chassi passou por uma análise em elementos finitos usando o SolidWorks, a fim de determinar sua rigidez torcional, caso não estivesse de acordo com a Tabela 1, o modelo seria revisado a fim de reforçá-lo e permitir seu enquadramento.

A análise ocorreu seguindo as mesmas configurações adotadas por Weiss (2016), fixando-se os pontos do amortecedor traseiro, e aplicando um binário de magnitude conhecida, nos pontos dos amortecedores dianteiros. Já que assim, garante-se que o momento gerado pelo binário atuasse longitudinalmente no chassi. Além disso, estas cargas devem ser aplicadas onde realmente haverá atuação de esforços no chassis durante seu uso, que seriam justamente nestes pontos (ver Figura 6), onde os amortecedores da suspensão transferirão cargas oriundas de seu trabalho para o chassis. Esta configuração, representa um caso comum de um veículo entrando em um obstáculo com apenas uma das rodas.

Figura 6:(roxo) cargas aplicadas e fixações (verde)

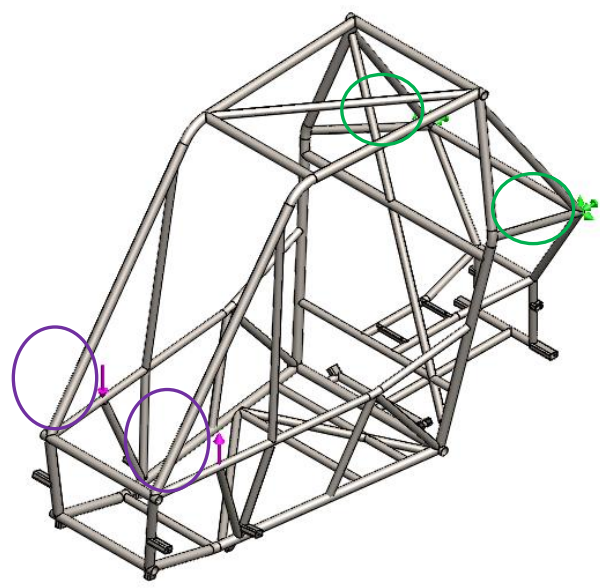




\section{RESULTADOS E DISCUSSÃO}

Após o término da simulação, foram obtidos os seguintes resultados:

Figura 7: Deslocamento vertical de acordo com simulação
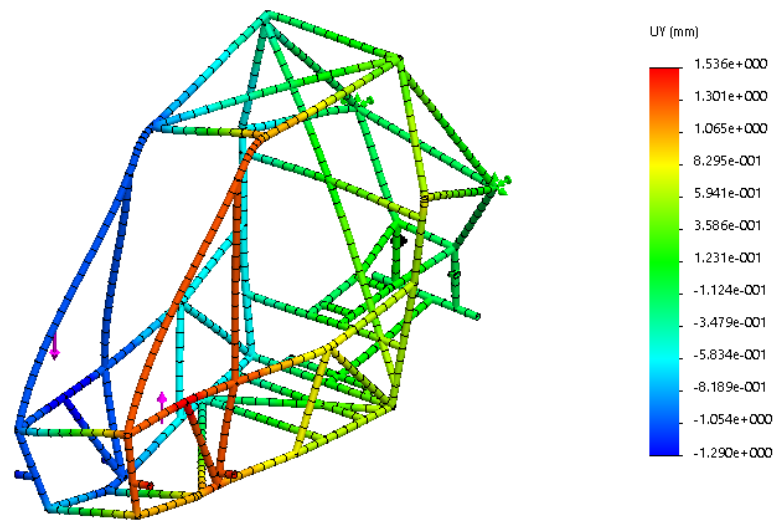

Tabela 2:Resultados das simulações variando a carga

\begin{tabular}{ccccccc}
\hline $\begin{array}{c}\text { Fator de } \\
\text { Aumento }\end{array}$ & Torque Aplicado (Nm) & $\begin{array}{c}\text { Deslocamento } \\
\text { Vertical }(\mathrm{mm})\end{array}$ & $\begin{array}{c}\text { Kt } \\
(\mathrm{Nm} / \mathrm{grau})\end{array}$ & Erro & Média & $\begin{array}{c}\text { Desvio } \\
\text { Padrão }\end{array}$ \\
\cline { 1 - 5 } & 400 & 2,8260 & 988,1471 & - & & \\
\hline 2 & 800 & 5,6520 & 988,1224 & 0,0247 & 988,1169 & 0,0271 \\
\cline { 1 - 4 } & 1200 & 8,4780 & 988,0813 & 0,0411 & & \\
\hline
\end{tabular}

Com essas modificações, o subsistema eliminou 3,3 metros de tubos de aço SAE 1020 de 1" da estrutura, que resultou em um chassi com uma massa de 3,92 kg mais leve. Ademais, o coeficiente de segurança no ponto mais solicitado, aplicandose 1200 N.m de carga nos pontos de fixação dos amortecedores dianteiros foi de 2,7. Mostrando que, não só a estrutura se apresenta rígida, como também está segura.

\section{CONSIDERAÇÕES FINAIS}

Através das análises obtidas nas simulações estruturais, pode-se entender a influência desta ferramenta no projeto de um chassi, pois esta permite ao projetista avaliar a estrutura rapidamente e, caso necessário, realizar modificações necessárias, garantindo uma maior previsibilidade do comportamento da estrutura quando for construída. 
Obviamente, por se tratar de um método numérico, com suas devidas simplificações - tanto no método, como em questões de software e possíveis erros de manufatura - há sempre um erro associado, exigindo que se realize, posteriormente, um ensaio com o modelo real, porém, estes resultados são precisos o suficiente para o projetista se basear durante o projeto em si.

A rigidez torcional do chassi se apresentou satisfatória - baseando-se nos dados da Tabela 1 - além disso, a estrutura respondeu aos esforços de maneira segura, baseando-se no coeficiente de segurança. Estes resultados permitem concluir que esta estrutura está apta a ser construída, e não será necessário fazer modificações estruturais.

\section{REFERÊNCIAS}

1SAMPÒ, Enrico. Modelling chassis flexibility in vehicle dynamics simulation. 2011. 185 f. Tese (Doutorado) - Curso de Engenharia, Faculty of Engineering and Physical Sciences, University of Surrey, Guildford, 2011.

${ }^{2}$ TEBBY, S. Methods to Determine Torsion Stiffness in an Automotive Chassis. Computer-aided Design and Applications, [s.I.], v. 8, n., p.67-75, 1 dez. 2011. CAD Solutions, LLC. http://dx.doi.org/10.3722/cadaps.2011.pace.67-75.

3BARBOSA, Luis Felipe Ferreira Motta. AVALIAÇÃO DA RIGIDEZ TORCIONAL DO CHASSI DE UM PROTÓTIPO BAJA SAE ATRAVÉS DO MÉTODO DE ELEMENTOS FINITOS E DE ENSAIO EXPERIMENTAL. 2010. 97 f. TCC (Graduação) - Curso de Engenharia Mecânica, Faculdade de Engenharia do Campus de Guaratinguetá, Universidade Estadual Paulista, Guaratinguetá, 2015.

${ }^{4}$ NORTON, Robert L. Projeto de Máquinas: Uma Abordagem Integrada. 4. ed. Worcester: Bookman, 2013. 1055 p.

5SAE BRASIL. RATBSB 2: REGULAMENTO ADMINISTRATIVO E TÉCNICO BAJA SAE BRASIL. 2 ed. S.I.: Baja Sae Brasil, 2018. 137 p.

'WEISS, Gabriel Hecker Evangelho. ANÁLISE COMPUTACIONAL E EXPERIMENTAL DE RIGIDEZ À TORÇÃO DE UM CHASSI DE FÓRMULA SAE. 2016. 85 f. Tese (Doutorado) - Curso de Engenharia Mecânica, Escola Politécnica, Universidade Federal do Rio de Janeiro, Rio de Janeiro, 2016.

7SILVA, Átila Paulino Corrêa da; VIEIRA, Paulo Henrique; TAVARES, Thomas. PROPOSTA DE OTIMIZAÇÃO ESTRUTURAL DO VEÍCULO BAJA SAE. 2014. 72 f. TCC (Graduação) - Curso de Mecânica Automotiva, Faculdade de Tecnologia de Santo André, Santo André, 2016.

${ }^{8}$ HIBBELER, R. C. Resistência dos Materiais. 7. ed. São Paulo: Pearson Petience Hall, 2010. 659 p. 